\title{
Human aging alters the neural computation and representation of space
}

\author{
Nicolas W. Schuck ${ }^{\mathrm{a}, \mathrm{b}, \mathrm{c}, *}$, Christian F. Doeller ${ }^{\mathrm{d}}$, Thad A. Polk ${ }^{\mathrm{e}}$, Ulman Lindenberger ${ }^{\mathrm{a}}$, Shu-Chen $\mathrm{Li}^{\mathrm{a}, \mathrm{f}}$ \\ a Max Planck Institute for Human Development, Center for Lifespan Psychology, 14195 Berlin, Germany \\ b Princeton University, Princeton Neuroscience Institute, Princeton, NJ 08544, USA \\ c Humboldt-Universität zu Berlin, Department of Psychology, 10099 Berlin, Germany \\ d Radboud University, Donders Institute for Brain, Cognition and Behaviour, 6525 Nijmegen, The Netherlands \\ e University of Michigan, Department of Psychology, Ann Arbor, MI 48109, USA \\ ${ }^{\mathrm{f}}$ TU Dresden, Department of Psychology, Chair of Lifespan Developmental Neuroscience, 01062 Dresden, Germany
}

\section{A R T I C L E I N F O}

\section{Article history:}

Received 22 October 2014

Accepted 13 May 2015

Available online 20 May 2015

\section{Keywords:}

Hippocampus

Striatum

Aging

Memory

Spatial navigation

Place cells

\begin{abstract}
A B S T R A C T
The hippocampus and striatum are core neural circuits involved in spatial learning and memory. Although both neural systems support spatial navigation, experimental and theoretical evidence indicate that they play different roles. In particular, whereas hippocampal place cells generate allocentric neural representations of space that are sensitive to geometric information, striatum-dependent learning is influenced by local landmarks. How human aging affects these different neural representations, however, is still not well understood. In this paper, we combined virtual reality, computational modeling, and neuroimaging to investigate the effects of age upon the neural computation and representation of space in humans. We manipulated the geometry and local landmarks of a virtual environment and examined the effects on memory performance and brain activity during spatial learning. In younger adults, both behavior and brain activity in the medial-temporal lobe were consistent with predictions of a computational model of hippocampus-dependent boundary processing. In contrast, older adults' behavior and medial-temporal lobe activity were primarily influenced by local cue information, and spatial learning was more associated with activity in the caudate nucleus rather than the hippocampus. Together these results point to altered spatial representations and information processing in the hippocampal-striatal circuitry with advancing adult age, which may contribute to spatial learning and memory deficits associated with normal and pathological aging.
\end{abstract}

(c) 2015 Elsevier Inc. All rights reserved.

\section{Introduction}

Many daily activities require memory-guided navigation in space. While human aging is associated with a deterioration of such abilities (Moffat, 2009), the precise changes in neural and cognitive processes are not well understood. The present study investigated age-related differences in the neural computations underlying spatial navigation.

Research on young animals has yielded much insight into the neural computations that underlie representations of space. Most strikingly, past studies of hippocampal subfields CA1 and CA3 have identified "place cells", which signal the position of the animal in the local environment (O'Keefe and Dostrovsky, 1971). Because the activity of these cells is related to memory functions, they have been proposed to form a so-called "cognitive map" (O'Keefe and Nadel, 1978), an allocentric representation of space that is independent of egocentric reference frames. While the computations that implement and update the representations of locations include factors insensitive to external stimuli

\footnotetext{
* Corresponding author at: Princeton Neuroscience Institute, Princeton University, Princeton, NJ 08544, USA.

E-mail address: nschuck@princeton.edu (N.W. Schuck).
}

(e.g., self-motion is used to perform path integration), place fields also systematically respond to changes in the environment (Muller and Kubie, 1987; O'Keefe and Burgess, 1996). Specifically, when the geometric parameters (e.g., length, width or shape of surrounding boundaries) of a familiar environment are changed, place cells are tuned to respond to the distance to the boundary (O'Keefe and Burgess, 1996). This influence of environmental geometry on neural firing patterns likely reflects the involvement of boundary-sensitive cells in the subiculum (Lever et al., 2009) and entorhinal cortex (Solstad et al., 2008). Place field activity has also been shown to relate directly to spatial memory (O'Keefe and Speakman, 1987), suggesting that hippocampusdependent memory is influenced by spatial boundaries.

In humans, behavioral studies have shown that spatial learning also shows similar sensitivity to boundaries, suggesting that the place field changes affect memory (Hartley et al., 2004; Doeller and Burgess, 2008). In addition, hippocampal blood-oxygen level dependent (BOLD) signals during both spatial navigation (Doeller et al., 2008) and spatial imagination in younger adults (Bird et al., 2010) are sensitive to boundary-related information, such that more boundary processing is associated with greater hippocampal activity. Given the importance of boundary information, and the known age-related decline in 
hippocampal function (e.g., Morcom et al., 2003), we investigated agerelated changes in the processing of boundary information in the hippocampal formation in younger and older adults.

Despite its crucial importance (Morris et al., 1982), the allocentric "cognitive map" in the hippocampal formation described above is not the only representation involved in spatial memory. Animal studies employing hippocampal lesions and other techniques have shown that navigation can also be based on associative learning processes (Tolman et al., 1946; Blodgett and McCutchan, 1947; Restle, 1957) that depend on the striatum (Packard and McGaugh, 1992; Devan et al., 1996). Although some research has characterized these associations as response learning (Packard and McGaugh, 1996), the striatum has also been shown to support response-independent navigation based on learning locations relative to visual cues (Packard et al., 1989; Packard and McGaugh, 1992; McDonald and White, 1994). In line with these findings in rodents, Doeller et al. (2008) showed that learning locations relative to a distinct visual cue ("landmark") in humans was associated with caudate activity and was consistent with predictions of a reinforcement learning model (Doeller and Burgess, 2008). Accordingly, we also studied age-related changes in striatumbased mechanisms of spatial memory, which in younger adults have been shown to be sensitive to the presence of visual cues that signal the target location in a particular direction and distance, rather than allocentric representations of space that involve information about the boundary.

While age-related volumetric decline (Raz et al., 2005) and impairments in memory functions suggest an impairment in hippocampus as well as striatum-dependent cognitive functions (e.g., Newman and Kaszniak, 2000; Morcom et al., 2003; Eppinger et al., 2013; Schuck et al., 2013b), investigations of age effects on spatial navigation have indicated a more nuanced pattern. Specifically, research has shown that aging impairs more recent but not remote spatial memory (Rosenbaum et al., 2012), and is associated with difficulties in switching to an allocentric spatial strategy (Harris et al., 2012; Wiener et al., 2013) as well as higher prevalence of egocentric strategies (Rodgers et al., 2012). Importantly, several studies have shown that extrahippocampal navigational strategies (Harris et al., 2012; Wiener et al., 2013), including landmark-based learning (Schuck et al., 2013a), seem to play a relatively larger role for spatial navigation in older adults. In line with this picture, neuroimaging studies of spatial navigation have found less hippocampal activation (Moffat et al., 2006) in older adults, and other studies have suggested that hippocampal volume is correlated with memory performance in old age (Konishi and Bohbot, 2013; Daugherty et al., 2014). In short, this evidence suggests that hippocampus-dependent spatial memory abilities are more strongly affected by age, potentially leading to older adults' heavier reliance on striatum-based strategies (see also, Moffat et al., 2007).

On the other hand, very little is known about how aging affects landmark and boundary processes specifically. We therefore examined agerelated differences in behavioral and brain responses related to processing boundary and landmark information, which are associated with hippocampal and striatal representations, respectively. To this end, we derived a computational model of boundary processing from a known model of place cell firing (Burgess and O'Keefe, 1996). We then applied this boundary model as well as a simple model of landmark processing (Doeller et al., 2008) to behavioral data obtained from a virtual environment experiment. These models enabled us to extract quantitative predictions of hippocampus-related boundary processing and striatumdependent landmark processing based on behavioral data. We then used these quantitative predictions to delineate brain activities related to boundary and landmark processing, respectively.

Based on the known age-related changes in hippocampal place cells (Barnes et al., 1997; Wilson et al., 2003), as well as our own previous work (Schuck et al., 2013a), we expected greater differences between age groups in hippocampus-based spatial memory than in striatalbased spatial memory. With respect to the computational modeling approach, we expected the hippocampus-based boundary model to account for younger adults' navigation better than that of older adults. In contrast, we expected the striatum-based landmark model to account for the behavior of older participants at least as well as that of the young, if not better. At the neural level, we expected greater hippocampal involvement in younger adults as well as an association between hippocampal activity and the behavior predicted by the boundary model. In older adults, we expected less hippocampal activity (Moffat et al., 2006) during spatial navigation. We also expected a correlation between caudate activity and landmark-based spatial memory. Finally, in line with earlier findings (Rieckmann et al., 2010; Schuck, 2013), we predicted that hippocampal function in older adults may actually contribute to cognitive abilities that are related to the striatum in younger adults.

\section{Materials and methods}

\section{Participants}

Participants were recruited from the existing database at the Max Planck Institute and were mostly local residents of Berlin-Wilmersdorf or Berlin-Dahlem (older adults) or students of Free University Berlin (younger adults). Potential participants were screened for health status with a verbal self-report questionnaire. Participants who reported subjective memory complaints or a history of neurological, cardiac or other significant physical diseases (including severe diabetes) were not included in the study. Twenty-eight younger (21-34 years, $m=28.1$ ) and 22 older (56-74 years, $m=67.2$ ) male adults completed the experiment (see Table 1 for sample statistics).

The local institutional review board approved the study and all participants provided informed consent. We included only male adults in order to avoid potential effects of sex on performance. One younger adult was excluded because his mean performance was more than $3 \mathrm{SD}$ from the group mean. Data from another young participant was excluded because of extensive signal drop in the MRI volumes, resulting in an overall sample of 26 younger and 22 older participants. Sample statistics including handedness, significant navigation experience ("Have you at any point in your life completed/worked on navigation tasks on a regular basis (sailing, airplane navigation, PC games, etc.)?" [yes/no]), health status and visual working memory performance are provided in Table 1.

The performance levels of our young and old samples in a visual working memory measure are comparable to previous studies ( $\mathrm{Li}$ et al., 2008). A linear regression including age and other potential covariates (i.e., self rated navigation experience, visual-spatial working memory and prior experience with Joystick handling) as regressors showed that none of these factors significantly accounted for navigation performance (target distance in last trial) or the landmark/boundary bias (difference in angle, see below) above the effect of age (all $p s>.10$ ).

\section{Virtual reality task}

The virtual reality task was programmed using UnrealEngine2 Runtime software (Epic Games; http://udn.epicgames.com/). The unit

Table 1

Average characteristics of the younger and older participants. Standard deviations are in parentheses.

\begin{tabular}{lll}
\hline & Younger adults & Older adults \\
\hline Age* $^{*}$ & $28.1(3.9)$ & $67.2(3.9)$ \\
Years of education & $12.1(1.0)$ & $12.0(1.4)$ \\
Handedness & R: $26, \mathrm{~L}: 0$ & $\mathrm{R}: 21, \mathrm{~L}: 1$ \\
Health, self report (1 = very bad, 7 = very good) & $6.1(1.0)$ & $5.6(1.0)$ \\
Visual working memory (2-back), \% correct* & $83.7(8.3)$ & $77.6(7.3)$ \\
Navigation experience, self report \% yes & 29.5 & 9.6 \\
Average in scanner movement $(\mathrm{x} / \mathrm{y} / \mathrm{z}, \mathrm{in} \mathrm{mm})$ & $0.3 / 0.5 / 0.6$ & $0.8 / 1.0 / 1.6$ \\
\hline
\end{tabular}

Note: * indicates a significant difference between age groups. 
for all coordinates is the virtual meter ( $1 \mathrm{vm}=62.5$ Unreal Units). Participants navigated in a first person view within a 3-dimensional virtual environment (VE) as shown in Fig. 1A. While participants were lying in the MRI scanner, the VE was projected using a back projection system with a set of mirrors. Navigation was controlled using a MR-compatible joystick (NAtA Technology, Coquitlam, Canada). The environment consisted of a grass plane surrounded by a circular stonewall (i.e., the boundary; diameter: $180 \mathrm{vm}$ ), a traffic cone (a landmark) and distal orientation cues (landscape of mountains, clouds, and the sun; projected at infinity, see Schuck et al. (2013a)). Walking speed was constant and allowed the participant to cross the entire arena in about $15 \mathrm{~s}$. Coordinates of the present location within the VE were recorded every $100 \mathrm{~ms}$.

Participants learned the locations of five everyday items in the VE (see Fig. 1B). The used objects were randomized between participants and a new set of objects was used in each run. The experiment was structured into two runs and followed procedures from our previous research (Schuck et al., 2013a). Each run included three trial types (Fig. 1B): In encoding trials, participants were instructed to pick up an object within the environment and remember the location of that object. At the start of each encoding trial participants were positioned close to the center of the environment and one object was placed within the environment. Once participants felt confident about their knowledge of the location, they could complete the trial by walking over the object (mimicking the act of picking it up). In feedback trials, each object was shown six times at its correct location (intermixed object order, see below and Fig. 1B). A feedback trial started with a picture (cue) of one object that was displayed on a gray background in front of the environment for $4 \mathrm{~s}$ and participants were instructed to navigate to the location where they remembered the cued object being located. Once a

\section{A: Procedure}

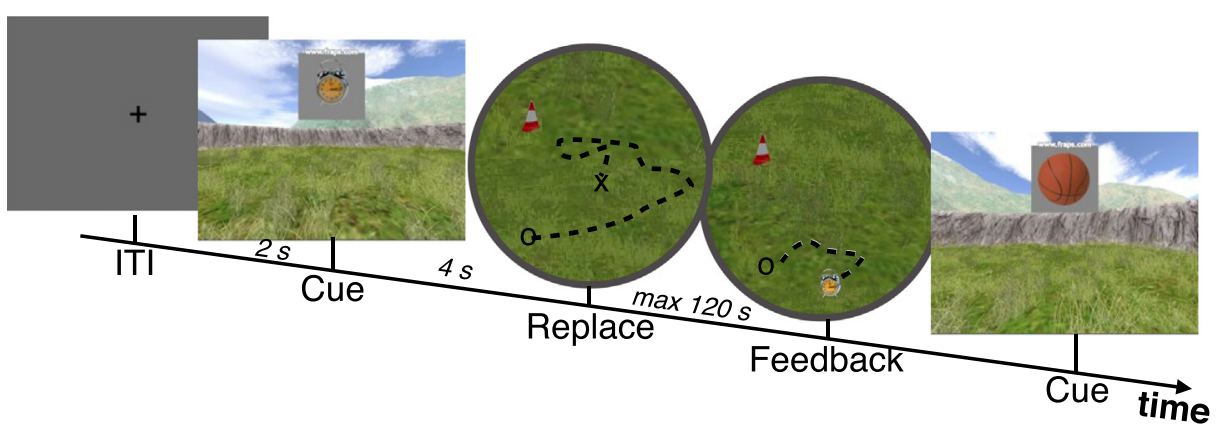

\section{B: Design}

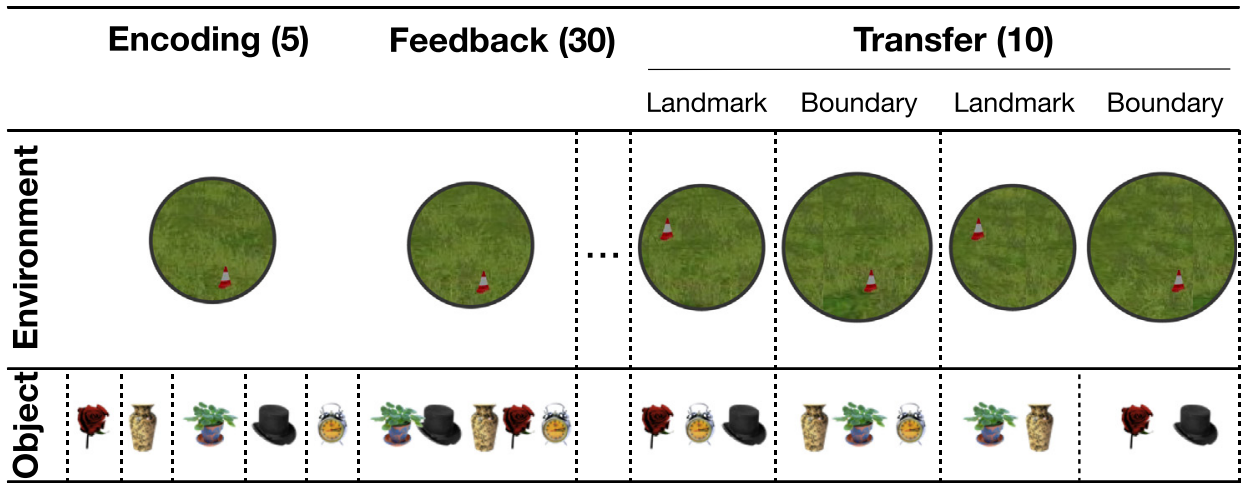

\section{C: Place Cell Model}

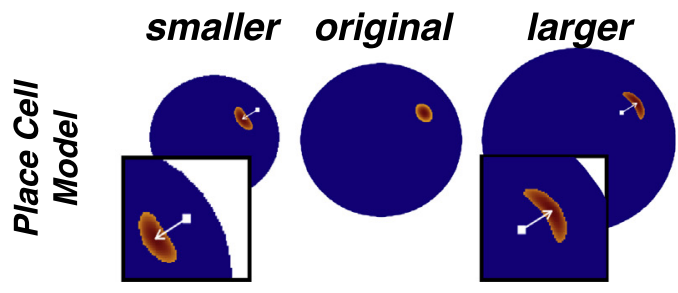

\section{D: Model-Based Analysis}

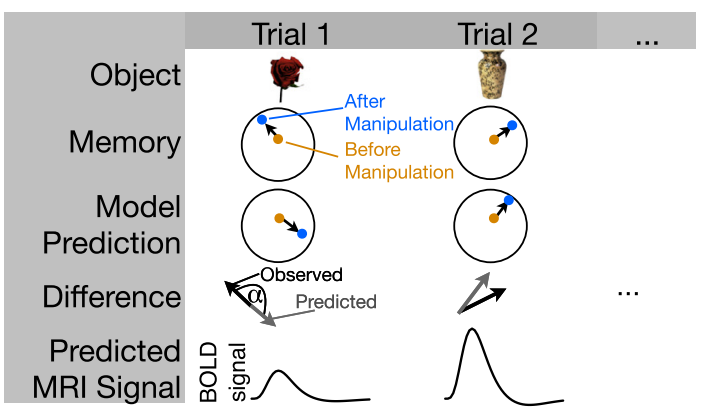

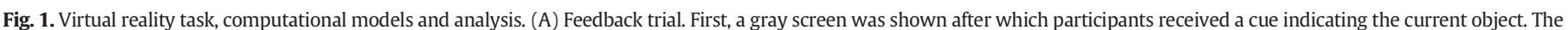

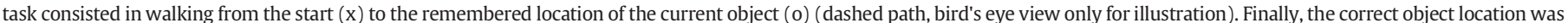

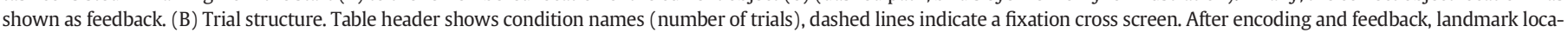

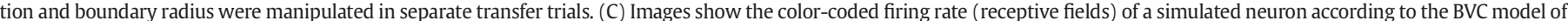

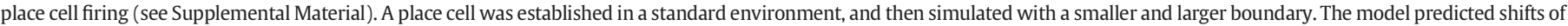

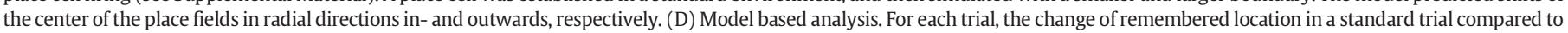

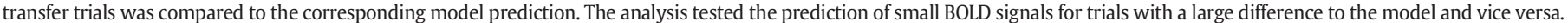


participant indicated per button-press that s/he had navigated to the position of her/his choice (memory), the object appeared at its correct location. Participants then saw how far their memory (current location) was from the correct answer (object location) and could use this information as feedback. Participants finally had to pick up the shown object and the next trial started from this pickup location. The learning (feedback phase) was followed by a transfer phase. In these trials, participants started either in an environment with a larger or smaller boundary ( $\pm 20 \%$ of original size $)$ or in an environment with the same boundary size but a changed landmark location ( $31.7 \mathrm{vm}$ from the original location, landmark location remained the same throughout the transfer phase). No feedback was given and the order of boundary and landmark trials was randomized between runs and participants, with two or three trials of one condition in a row separated by a fixation screen. Overall each object appeared in each of the two conditions once, resulting in ten transfer trials overall.

One run contained the smaller boundary condition, and one run contained the larger boundary condition. Participants were instructed about the general purpose of the experiment (learning and remembering the locations of objects), but were naïve to the boundary/landmark manipulations. All trials had a maximum search time of $120 \mathrm{~s}$ (mean younger: 18.9 s, $1.1 \%$ timeouts; mean older: 21.5 s, $1.5 \%$ timeouts). Immediately before the start of scanning, joystick-handling was practiced outside as well as inside the MRI scanner. Additionally, the procedures of the task were trained outside of the scanner using a regular joystick in an unrelated VE and under the guidance of the experimenter for about $15 \mathrm{~min}$. Virtual reality technology has been used extensively in previous research with humans (e.g., Moffat and Resnick, 2002; Doeller et al., 2010; Etchamendy et al., 2011) as well as with animals (Dombeck et al., 2010; Chen et al., 2013; Domnisoru et al., 2013; Schmidt-Hieber and Häusser, 2013). This research has shown that despite the differences between virtual and real spatial navigation (e.g., proprioception), results are largely comparable.

\section{Image acquisition}

We used a 3 Tesla Siemens Magnetom Trio (Siemens, Erlangen, Germany) research-dedicated MRI scanner to acquire all the data. At the start and end of functional scanning, T1-weighted structural images were acquired with an MP-RAGE pulse sequence with a resolution of $1 \mathrm{~mm}^{3}$ $(\mathrm{TR}=2500 \mathrm{~ms}, \mathrm{TE}=4.77 \mathrm{~ms}, \mathrm{TI}=1100 \mathrm{~ms}$, acquisition matrix $=$ $256 \times 256 \times 192$, FOV $=256 \mathrm{~mm}$, flip angle $=7^{\circ}$, bandwidth $=$ $140 \mathrm{~Hz} / \mathrm{Px})$. Functional data were acquired using a T2*-weighted echo-planar imaging (EPI) pulse sequence $(3 \times 3 \times 3 \mathrm{~mm}$ voxels, slice thickness $=2.5 \mathrm{~mm}$, distance factor $=20 \%$, TR $=2400 \mathrm{~ms}$, TE $=$ $30 \mathrm{~ms}$, image matrix $=72 \times 72, \mathrm{FOV}=216 \mathrm{~mm}$, flip angle $=80^{\circ}, 43$ axial slices, GRAPPA parallel imaging, acceleration factor: 2 , interleaved acquisition). To reduce signal drop-out in medial temporal areas, EPI slices were acquired that were rotated about $-30^{\circ}$ relative to the anterior-posterior commissure axis. The EPI sequence provided coverage of most brain areas ( $\sim 5,000$ voxels, some loss in superior parietal cortex and cerebellum caused by slice tilt, see Supplemental Material, Figure S2). The task was split into two functional runs, which lasted between 10 and $40 \mathrm{~min}$, depending on the time it took of each participant to replace/collect all objects. Between the two functional runs, field maps for distortion correction were acquired using an EPI sequence (TEs $=4.92$ and $7.38 \mathrm{~ms}$, TR $=488 \mathrm{~ms}$, matrix size $64 \times 52,43$ slices, voxel size $=4 \times 4 \times 3 \mathrm{~mm}$ ). To allow for equilibration effects, the task started after four volumes.

\section{Behavioral analysis}

All behavioral analyses were done using $\mathrm{R}$ ( $\mathrm{R}$ Development Core Team, 2011). We analyzed object location memory during the feedback phase by calculating the Euclidean distance between the remembered and the correct locations. A baseline (dashed line in Fig. 2A) was calculated by simulating $10^{5}$ uniformly distributed points. For the data from the transfer phase, we computed the vector that connected the remembered locations in the last trial before the transfer and the remembered location for the same object during the landmark and boundary transfer conditions. Then, we compared the angle of these vectors to the angle predicted by the different models (see below).

\section{Boundary model and landmark models}

The main goal of the models was to provide quantitative predictions of the expected behavior resulting from boundary and landmark sensitivity. The presented boundary model is derived from a model that was proposed to account for the behavior of place cells under conditions of changed geometry (Boundary Vector Cell (BVC) Model, O'Keefe and Burgess, 1996). Importantly, previous research has indicated that the modeled changes in place cell firing directly affect memorized locations (O'Keefe and Speakman, 1987; Hartley et al., 2004). In addition, it has been shown that boundary processing is related to greater hippocampal activity (Bird et al., 2010), suggesting that greater sensitivity to the boundary should be related to greater hippocampal activation. Hence the BVC model provides a useful tool to bridge the gap between insights on the cellular, behavioral and BOLD signal levels.

Using an adapted BVC model (see Supplementary material, Fig. S1), our simulations showed that, under conditions of changed geometry, previously established place fields changed their location such that the distances to nearby boundaries are approximately equal to those in the unchanged, familiar environment (Fig. 1C), consistent with the experimental results (Muller and Kubie, 1987; O'Keefe and Burgess, 1996). Hence, the central prediction is that the center of place fields should shift radially inwards for smaller environments and radially outwards for larger environments. Based on these results, we formulated a simpler model that captures the basic prediction (inward and outward shifts depending on boundary changes). We will call this the simplified geometric model (SGM) of boundary processing and test its predictions on the level of behavior and neuroimaging. This model corresponds to a simple geometric transformation of each point $\mathbf{p}$ to $\tilde{\mathbf{p}}_{m}$ according to the change in radius $\Delta r$ in a radial direction:

$\tilde{\mathbf{p}}_{m}=\left(1 \pm \frac{\Delta r}{r^{2}}|\mathbf{p}|\right) \mathbf{p}$.

In addition to the radial angles of the shifts, this model predicts that the size of the shift of a remembered location is zero if the remembered location is in the center of the environment and the size of the shift corresponds to the change of the radius $\Delta r$ if the place field is exactly at the edge of the environment. However, we do not see a firm justification for this assumption and hence proceeded to test only the predicted direction of the shift of remembered locations, but not the predicted size of the shift.

To quantify the behavior that would result from processing the landmark instead of the boundary cues, we calculated behavioral predictions for the landmark transfer trials as well. In particular, we assumed that landmark processing would result in shifting the remembered location in the same direction as the landmark was shifted (Doeller et al., 2008; Schuck et al., 2013a). This means that if the landmark in the transfer condition is shifted by a translation vector $v_{L M}$, the predicted remembered locations are shifted by the same vector.

To compare the model and empirical data from the transfer conditions, we first calculated the expected memory changes under both models as described above. As mentioned, each model makes a prediction of a memorized object location, which we term $\tilde{\mathbf{p}}_{m}$. In comparison to the same objects' original location (p), the models thus predict a direction in which the memory of an object location is shifted following our experimental manipulations of the boundary or landmark. Mathematically, this direction is the angle of the vector that connects the 
A

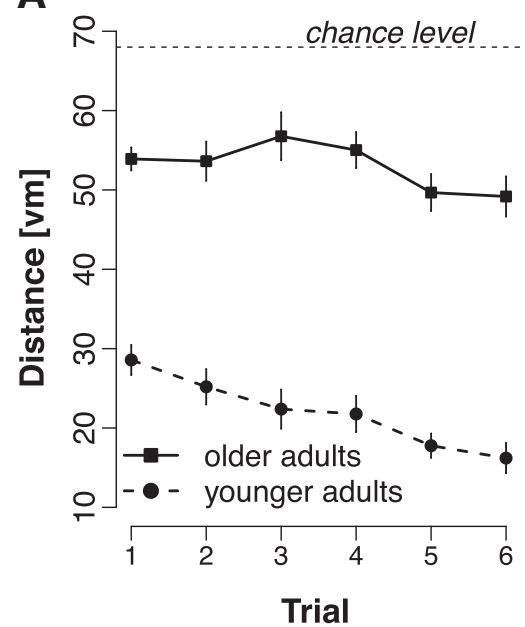

C

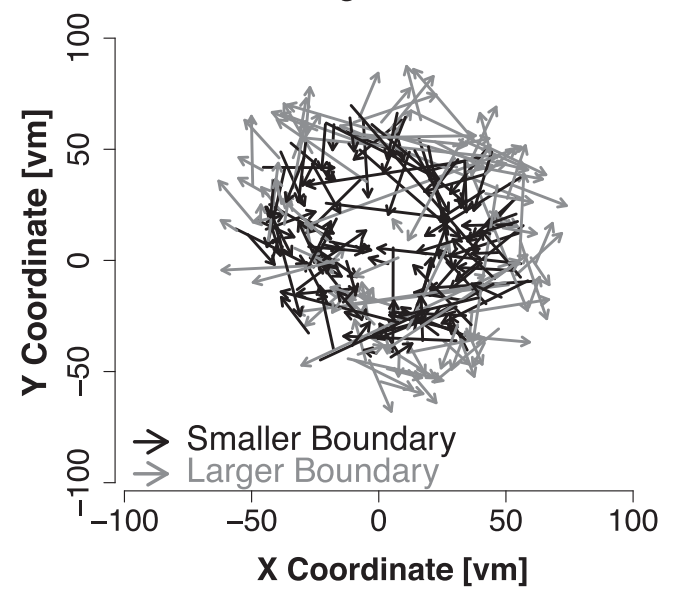

B

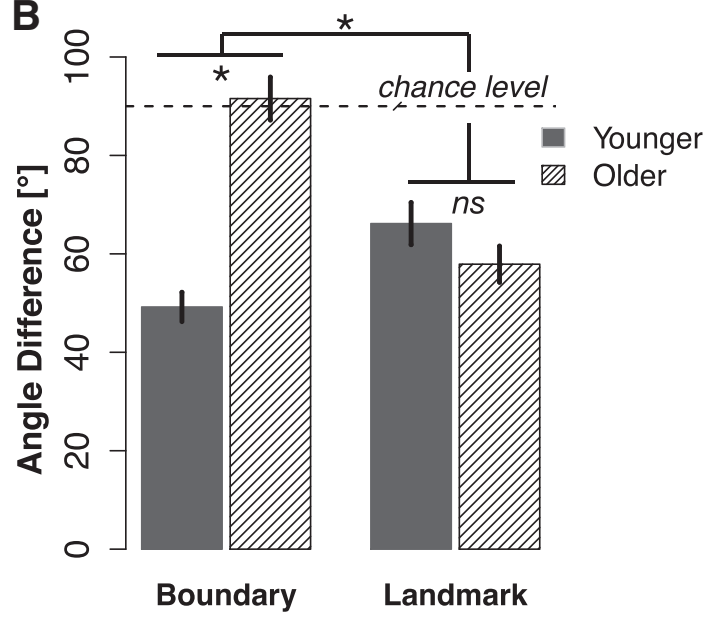

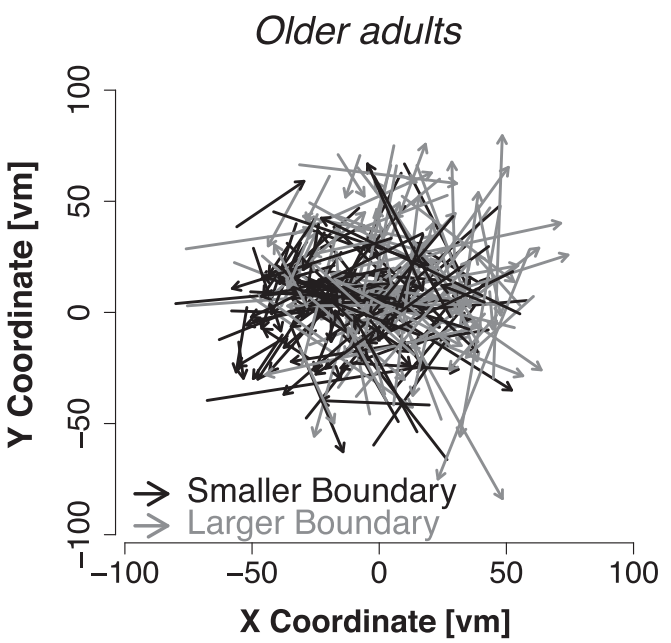

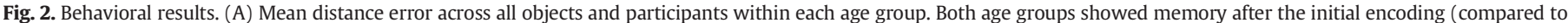

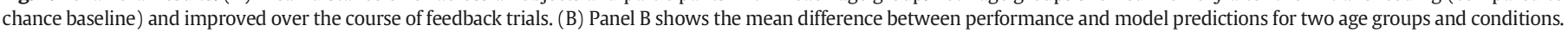

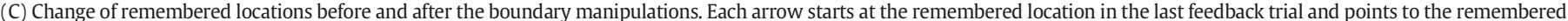

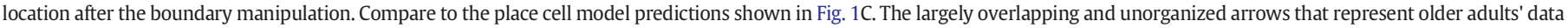
reflect the complete lack of boundary processing in this age group (see panel B). Error bars represent \pm 1 SEM. Stars indicate significance at $p<.05$.

original location $\mathbf{p}$ (the drop location in the last feedback trial) and the predicted transfer location $\tilde{\mathbf{p}}_{m}$. These direction changes are then compared to the observed direction changes, which can be measured as the angle of the vector that connects the original memory location $\mathbf{p}$ to the observed memory locations for the same object in the transfer trials, $\tilde{\mathbf{p}}_{0}$. Thus comparing the model's predicted angle of change $\left(\gamma_{m}\right)$ to the observed angle $\left(\gamma_{o}\right)$ can be achieved by taking the difference between the angles of the vectors $\left(\mathbf{p}-\tilde{\mathbf{p}}_{m}\right)$ and $\left(\mathbf{p}-\tilde{\mathbf{p}}_{o}\right)$ :

$\gamma_{m}-\gamma_{O}=\tan ^{-1}\left(\mathbf{p}-\tilde{\mathbf{p}}_{m}\right)-\tan ^{-1}\left(\mathbf{p}-\tilde{\mathbf{p}}_{o}\right)$

\section{fMRI analyses}

Imaging data was preprocessed and analyzed using SPM8. Functional volumes were distortion corrected utilizing field maps (Jezzard and Balaban, 1995) and spatially realigned using SPM's Unwarp routine (Andersson et al., 2001). Motion correction was done using 2nd degree interpolation, quality of 0.9 and FWHM of $4 \mathrm{~mm}$. Table 1 lists the average maximum displacement during scanning for younger and older adults. All analyses used SPM's implicit masking and considered only voxels present in all subjects. Fig. S2 in the Supplementary material shows the brain coverage for the second-level analyses.

Next, the mean of the realigned and corrected volumes were coregistered with the structural scan (reference: mean image, source: anatomical, cost function: normalized mutual information). Anatomical scans were then segmented using the New Segment procedure (Ashburner and Friston, 2005) using a regularization parameter of 4 . We used DARTEL to generate a study specific template (Ashburner and Friston, 2009) to avoid a bias towards younger participants' brain morphology. Supplemental Table S1 summarizes the preprocessing steps and parameters (Poldrack et al., 2008). Fig. S2 shows the covered area of the brain. Control analyses indicated that changed brain coverage did not affect our results (see Fig. S2). Images were spatially smoothed with an $8 \mathrm{~mm}$ Gaussian kernel. Anatomical ROIs were based on the Automated Anatomical Labeling atlas (Tzourio-Mazoyer et al., 2002). The centroid coordinates of the left and right hippocampi were $[-24,-22,-8]$ and $[28,-22,-10]$, respectively. Centroid coordinates of the left and right caudate nuclei were $[-12,8,8]$ and $[12,8$, 8], respectively (see Fig. 3).

We analyzed the data from the feedback phase using SPM8. We used a design matrix that included regressors for the display of a cue, the period between the cue display and the button press (replace), the 


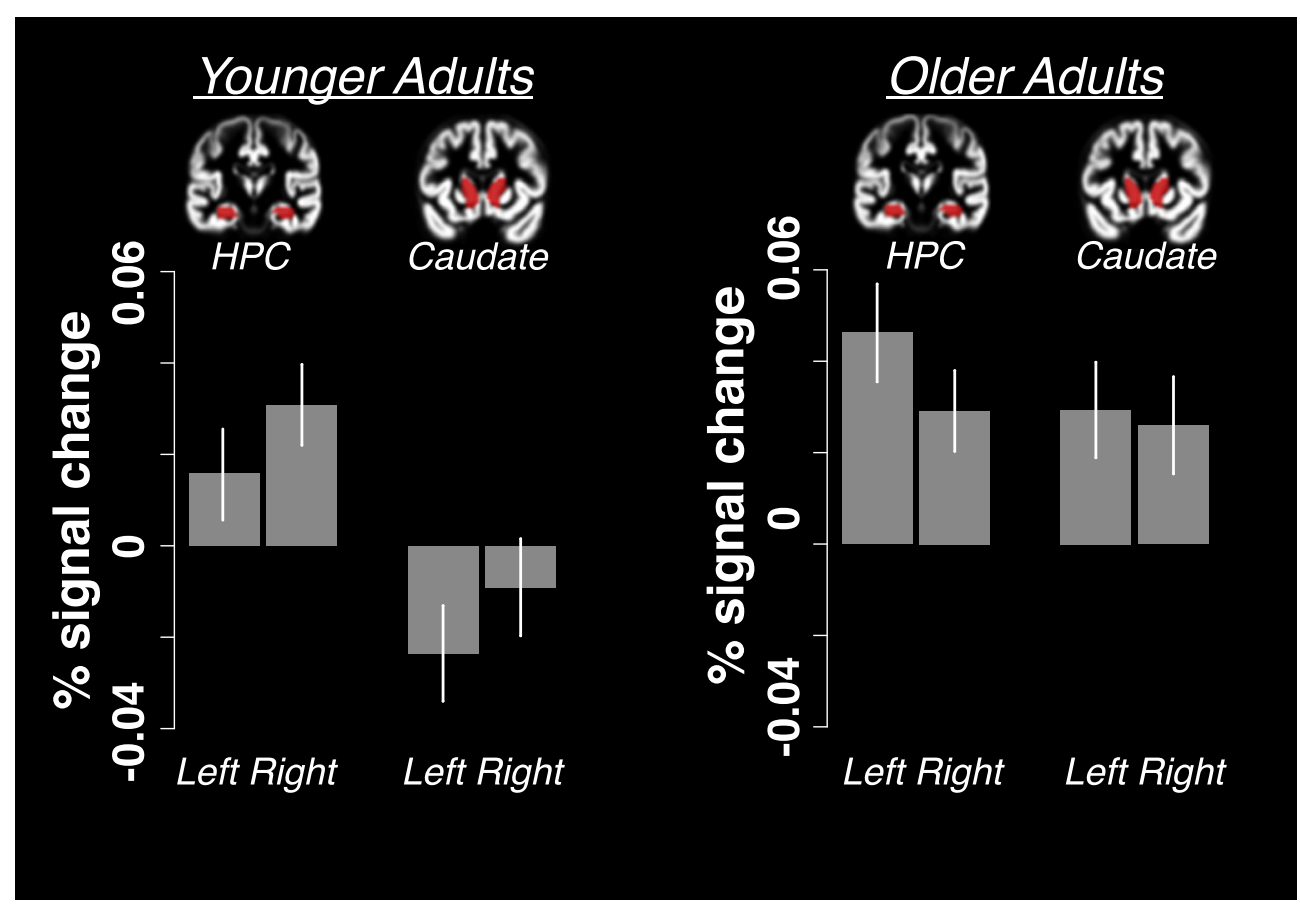

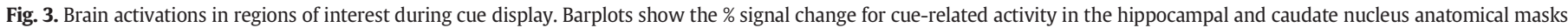
(based on AAL labels, see brain maps above bars). Error bars represent \pm SEM. Activations of other regions can be found in the Supplemental Table S2 and Fig. S3.

feedback display until feedback collection, the gray screen between blocks (ITI) and the motion parameters that resulted from motion correction. For the model-based analysis, we determined the mismatch between the model and the behavior for each object (see above, Eq. (2), one value for each object that was applied to each occurrence of this object) and used these as parametric modulators (see Fig. 1D). Given the role of reinforcement processes in landmark-dependent learning (Doeller and Burgess, 2008), the parametric modulators for the landmark model were applied to the feedback regressor, while the boundary model modulators were applied to the replace regressor. Hence this analysis tested the hypothesis of larger brain activity for objects that have less mismatch to the model predictions.

Individual contrasts were submitted to second-level ANOVA and between-group t-tests. The ANOVA included a covariate that reflects the performance difference between the landmark and boundary transfer trials (as shown in Fig. 2B). This covariate was centered separately with respect to the corresponding age group mean. The key results are unaffected by the inclusion or exclusion of this covariate. Unless specified otherwise, a voxel-level threshold of $p<.001$ was used with the appropriate cluster level correction as computed by the AFNI program 3dClustSim $(\mathrm{k}=19)$.

We also conducted a performance-group analysis by splitting younger and older adults based on their respective group's median performance in the feedback phase. Then, we extracted contrast estimates of the boundary and landmark models in a (para)hippocampal and a striatum ROI separately for high and low performing younger and older adults. The ROIs were defined based on a low threshold $(p<.005$, $\mathrm{k}=20$ ) group analysis that compared the effect of boundary and landmark regressors (same as in Fig. 4, only the threshold was lower): the hippocampus ROI was an $8 \mathrm{~mm}$ sphere around the hippocampal peak of activation in younger adults (Fig. 5A) and the striatal ROI was an $8 \mathrm{~mm}$ sphere centered on the striatum peak in the analysis of older adults (Fig. 5B). Because the model-based analysis was agnostic to inter-individual differences in overall performance level (a purely

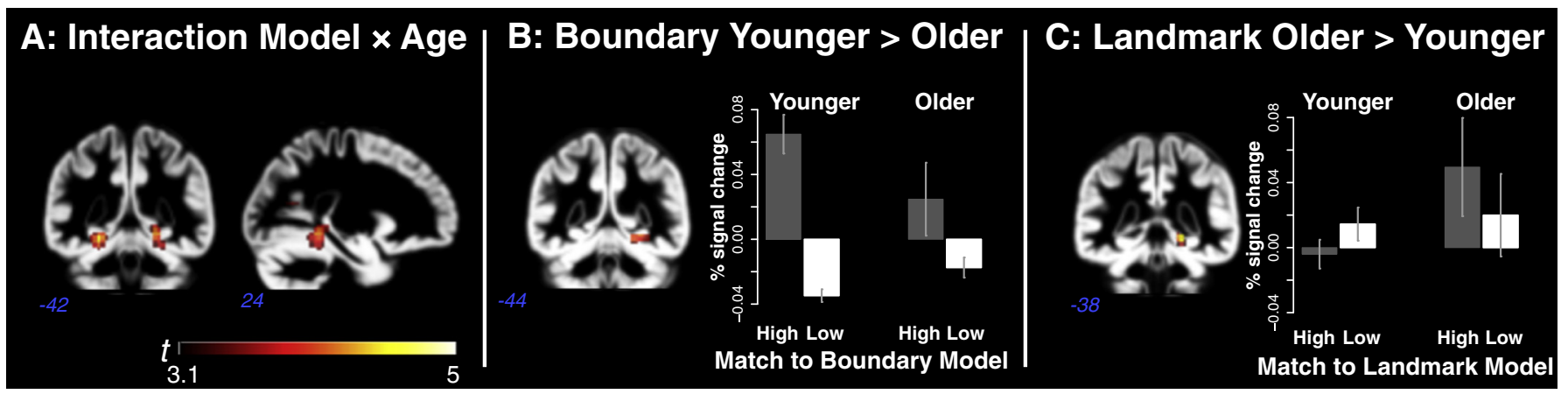

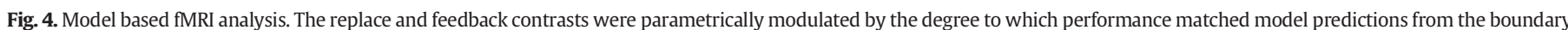

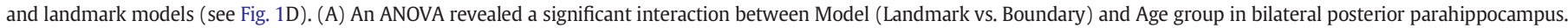

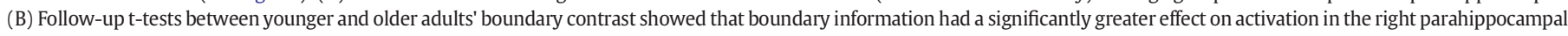

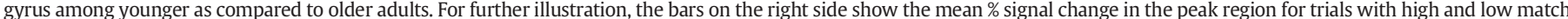

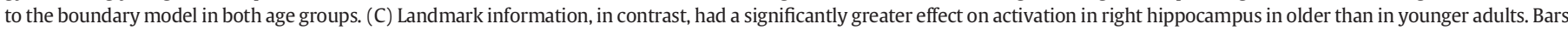

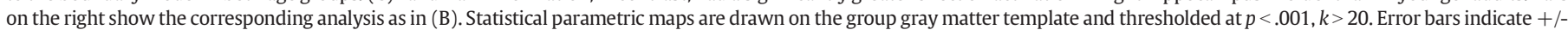
S.E.M. 


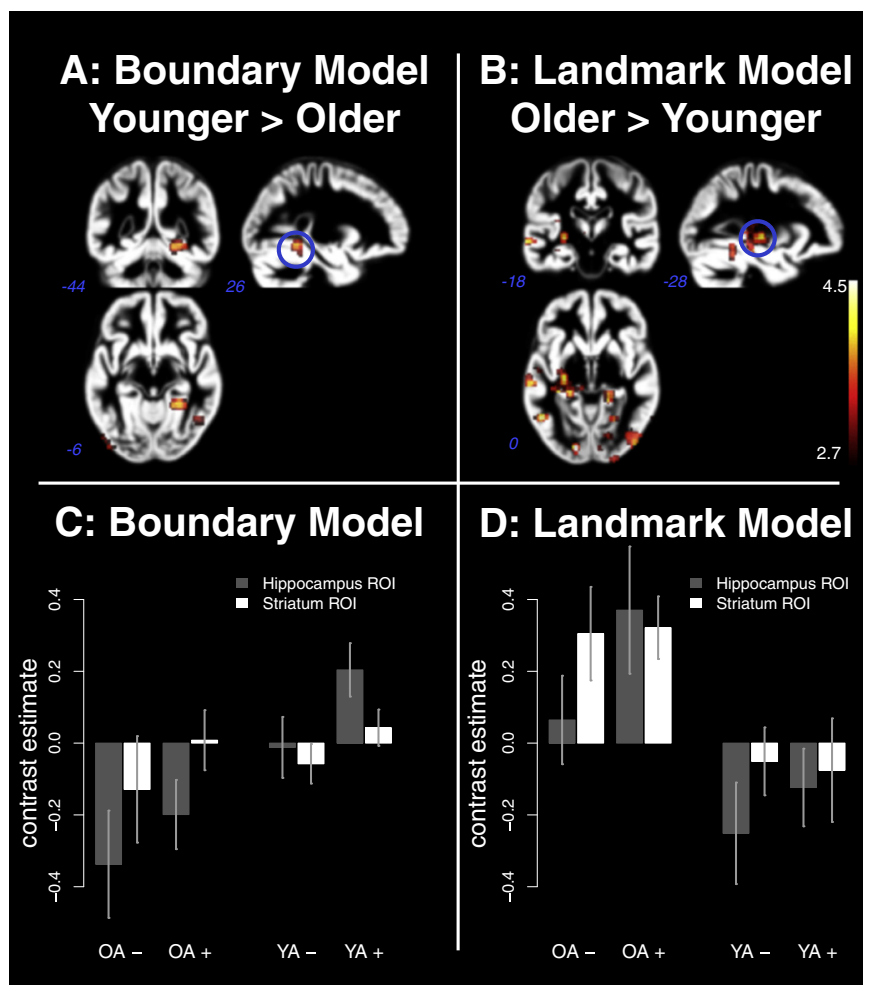

Fig. 5. Panels A and B: Model based fMRI analysis as in Fig. 4, except that the statistical threshold was set to $p<.005$, cluster size 20 voxels. At this less conservative threshold older adults showed an association between landmark model based behavior and Putamen activity. In younger adults, the effect of the boundary model remained essentially unchanged. Panels C and D: Mean contrast estimates for a (para-)hippocampal ROI ( $8 \mathrm{~mm}$ sphere around peak of activation in younger adults, see blue circle) and a striatal ROI ( $8 \mathrm{~mm}$ sphere, striatum peak in analysis of older adults, see blue circle) for the boundary and landmark models. The age groups are further split up by learning success, whereby "+" denotes participants that have above median learning (error in trial 1 minus error in trial 6), whereas a "-" denotes the corresponding low performance group. Error bars indicate +/- S.E.M.

within-subjects analysis for which all parametric regressors are normalized within subjects), a median split on between-subject performance differences provides unique information about the potential relations between the performance level and brain correlates (c.f., Chowdhury et al., 2013; Nagel et al., 2009).

\section{Results}

Age differences in spatial memory and sensitivity to boundary and landmark changes

Data from 26 younger $(21-34, m=28.1)$ and 22 older (56-74, $m=67.2$ ) male participants were analyzed (see Table 1 for sample statistics). Fig. 2A shows the mean distance between the remembered and the correct target locations as a function of trial (average across all objects). Younger adults performed significantly better than older adults, $F(1,46)=163.2, p<.001$. Moreover, both age groups improved over the course of the feedback trials, i.e. there was a linear trend of trial: $F(5,230)=32.63, p<.001$. There was also a significant age group $\times$ trial interaction, reflecting a larger reduction of distance error in younger than in older adults, $F(5,230)=4.61, p=.037$.

To analyze the sensitivity of each participant to boundary and landmark information, we calculated the difference between the angle predicted by the SGM (the simplified geometric model which captures the influence of boundary cues) and the observed angle (i.e., the angle of the vector connecting the remembered location before and after the boundary change) as well as the angle difference for the landmark transfer trials (Doeller et al., 2008). An ANOVA of these model fits revealed a main effect of age group, $F(1,46)=14.4, p<.001$ as well as an age group by model (Landmark vs. Boundary) interaction, $F(1$, $46)=53.9, p<.001$. Fig. 2B shows the match between the participants' behavior in the transfer trials and the predictions of the boundary and landmark models (difference between the predicted and observed angles, see Boundary model and landmark models). As can be seen, younger adults showed behavior that was consistent with the boundary model (compared to the chance baseline of $90^{\circ}$ ), $t(25)=-13.6, p=$ $<.001$. Younger adults also showed behavior consistent with landmark processing, $t(25)=-5.3, p<.001$, but this effect was smaller compared to boundary processing, $t(25)=-3.6, p=.005$. In contrast, older participants showed stronger indications of landmark as compared to boundary processing, $t(21)=7.2, p<.001$ (comparing the fits of the Landmark and Boundary models). Hence, compared to younger adults their behavior was less consistent with the boundary model, $t(37.93)=7.94, p<.001$, but did not differ with respect to the prediction of the landmark model, $t(45.82)=-1.67, p=.102$. Fig. $2 C$ illustrates the age differences in memory representations by showing the remembered locations during the boundary transfer condition (arrowhead) relative to the corresponding remembered locations before the boundary change (arrow tail). As can be seen, only younger but not older adults were sensitive to boundary change and shifted their remembered response locations radially in- or outwards depending on the direction of the boundary change (i.e., a smaller or larger boundary, respectively).

\section{Age differences in hippocampal and striatal contributions}

We first investigated task-related activations in the regions of interest, by contrasting brain activity during cue display against baseline (inter-trial interval) within anatomical ROIs of the hippocampus and the caudate (see fMRI analysis for details; masks based on AAL, see Tzourio-Mazoyer et al., 2002). Among younger adults, comparing ontask cue processing with baseline showed greater activation in the hippocampus ROI, but not in the caudate ROI. In older adults, caudate nuclei as well as hippocampi were positively activated during cue processing (see Fig. 3), resulting in a significant ROI by age group interaction (Interaction Age $\times$ Area: $F(1,44)=8.3, p=.006$, main effect Area: $F(1,44)=$ $16.9, p<.001$, Interaction Age $\times$ Laterality: $F(1,44)=15.9, p<.001$; all other effects n.s.). The results of a corresponding whole-brain analysis are reported in the Supplementary information (Table S2 and Fig. S3).

To test our main hypothesis and investigate age-related effects on boundary and landmark processing, we performed model-based analyses, in which we fitted one general linear model containing predictions of the boundary model and the landmark model to fMRI signal variations as described above. For each type of prediction (boundary or landmark), we included a separate parametric regressor that reflected degree of match between the model predictions and the observed behavior for each object on a trial-by-trial basis (Fig. 1D). An ANOVA with factors model (boundary vs. landmark) and age group (younger vs. older) revealed a significant interaction between these factors (see Fig. 4A) in bilateral (para)hippocampus (peaks at MNI coordinates $21 /-38 /-3$, and $-24 /-45 /-9)$, bilateral occipital cortex $(45 / 76 / 3$, $-21 / 86 / 17,13 / 80 / 5)$, calcarine gyrus $(17 /-59 / 15)$ and middle temporal gyrus $(-64 /-18 / 3)$. Tests for the main effects of age and model did not show any significant activation. A follow up t-test that directly compared the effect of the boundary regressor between the age groups revealed a single peak in the right posterior parahippocampal gyrus, extending into the hippocampus $(21 /-42 /-3$, Fig. 4B). With respect to the landmark model, we observed greater association of landmark model predictions with BOLD activity in the right hippocampus $(21 /-39 /-3$, Fig. 4C) in older compared to younger adults. All results were cluster corrected. 
To further investigate the role of the striatum in landmark based processing, we applied a more lenient threshold to the analysis of the landmark regressor ( $p<.005$, cluster size $=19$, see Figs. $5 A / B)$. This analysis showed additional activity for older adults in the thalamus and putamen (MNI: $-27 /-18 / 0$, see Fig. 5B), whereas a corresponding control analysis for the boundary model did not show such a pattern (no younger adults' boundary related activity in the striatum and overall more focal brain activation patterns).

Finally, we investigated the link between overall performance level and the recruitment of hippocampal and striatal activity in relation to the landmark and boundary models. To this end, we extracted contrast estimates of the boundary and landmark models in a (para)hippocampal and a striatum ROI separately for high and low performing younger and older adults (see fMRI analyses, and Fig. 5, panels $C$ and $\mathrm{D}$ ). We found that only high performing younger adults showed a positive contrast in the (para)hippocampus ROI, whereas the low-performing younger adults did not. Interestingly, for the landmark model, high-performing older adults showed additional activation in the hippocampus ROI in addition to the striatal activation, whereas low-performing older adults only showed activation in the striatum ROI. Hence, among older adults, additional hippocampus function for landmark learning was related to superior learning performance.

In summary, the above reported analyses showed greater activity in the hippocampus during cue display and an association between parahippocampus activity and the predictions of the boundary model. We also found greater activity in the caudate during cue processing in older adults. Interestingly, the model-based analyses revealed an association between higher hippocampus activity and landmark processing in older adults, consistent with the hypothesis that the hippocampus is involved in processing landmark but not boundary information in this age group.

\section{Discussion}

The present study investigated the effects of aging on the neural computations and representations underlying spatial navigation in human males. Based on data from studies with animals and younger adults, we distinguished between two processes: a hippocampal process that is sensitive to the distance to boundaries, and a caudate nucleus-based process that is sensitive to landmarks (Packard et al., 1989; Doeller and Burgess, 2008; Doeller et al., 2008). Using computational modeling, we derived quantitative predictions for spatial memory performance that reflect the influence of the landmark and boundary aspects of the environment (Doeller et al., 2008) and using a modelbased fMRI approach, we looked for brain areas in which the BOLD activity was greater when memory performance was more consistent with boundary processing or landmark processing.

Our model-based analyses showed that activity in the hippocampal formation in younger but not older adults was correlated with boundary processing. Older adults, in contrast, showed no indications of boundary-based spatial learning. Their spatial memory was, however, sensitive to landmark information and the model-based analysis revealed a link between older adults' behavior, predictions derived from the landmark model, and activity in the right hippocampus.

These results are significant from several perspectives. First, this is the first model-based fMRI study informed by animal and computational models of hippocampal place-cell functioning. The results from the younger participants are consistent with existing data from animal (O'Keefe and Burgess, 1996) and human (Hartley et al., 2004; Bird et al., 2010) research, but they strengthen and specify the role of boundary information in (para) hippocampal neural computations and memory performance in humans (Bird et al., 2010).

Second, these findings are the first demonstration of age-related impairments in the specific computations (boundary vs. landmark processing) and resulting representations (allocentric representations vs. representations reliant on discriminative cues/landmarks) during spatial navigation. Our analyses allowed us to relate adult age differences in spatial learning to the computational functions of hippocampal and striatal circuitry. By demonstrating differences in spatial representations between older and younger adults, we went beyond the results of previous studies utilizing VE tasks, which showed that older adults form degraded configural representations relative to younger adults (i.e., forming and using a cognitive map, see Iaria et al., 2009). It is important to keep in mind that our results are based only on male participants. While this approach avoided issues of gender effects in spatial navigation, it potentially limited the external validity to male humans. While replicating our own previous report of asymmetric age differences for landmark- and boundary-related learning (Schuck et al., 2013a), we also extended those results by using quantitative models and investigating the underlying neural mechanisms.

Third, and perhaps most striking, we found an association between landmark processing and hippocampal activity in older adults. The lack of evidence for a link between landmark-based navigation and striatal activity seems surprising. At the same time, it is in line with our previous report of an influence of the KIBRA gene on landmark processing in older adults (Schuck et al., 2013a,b): because the KIBRA gene is related to hippocampal processes (Schneider et al., 2010), this link supports our current findings of a relation between neural processes in the (para)hippocampus and landmark processes in older adults. Moreover, at a more lenient threshold, older adults did show landmark related activity in the striatum (see Fig. 5B). So the striatum may still be playing a role in landmark based processing in older adults, but this effect seems weak and requires further investigation. On a more general level, this finding supports the notion that the hippocampus plays an important role in age-related effects on spatial navigation (Moffat et al., 2006). It also suggests that the processes associated with hippocampal activity might be different between younger and older adults. In short, our findings reflect age-related changes in the distribution of spatial information processing across the brain and are potentially linked to declines in memory functions that rely on hippocampal (Moffat et al., 2006) and striatal (Eppinger et al., 2013) regions.

Despite the specificity of our findings from the model-based analysis, mean-based analyses of activity during cue display showed that older adults generally exhibited higher activation in a large cortical network (Fig. S3), a common finding in fMRI studies of aging (ReuterLorenz and Lustig, 2005). Moreover, our finding of altered brainbehavior relationships in older adults is consistent with several studies investigating brain activity patterns related to declarative and nondeclarative memory in older adults (Rieckmann et al., 2010). These studies reported hippocampal activity in older adults during tasks that primarily involve the striatum in younger adults. In particular, older adults exhibited simultaneous hippocampus and caudate activation during an implicit memory task that depends on the striatum in younger adults. Other studies investigating Huntington's patients (Voermans et al., 2004) also reported hippocampal hyperactivation during a memory task in patients vs. controls and lesion studies in animals suggest that striatal lesions may also result in changes in hippocampus depend memory or vice versa (for an overview, see Schuck, 2013). In the current study, the phenomenon of hippocampal hyperactivation was specifically related to spatial memory processes in older adults.

Two possible explanations, which are not mutually exclusive, can be offered to account for this finding. First, the additional hippocampal activity in older adults might be associated with better memory compared to their same aged peers. (Voermans et al., 2004; Rieckmann et al., 2010). This hypothesis predicts that better memory performance in a task that is primarily striatum-dependent in younger adults should be related to the additional recruitment of hippocampal activity in older adults. This prediction has been supported for striatum-dependent implicit learning (Rieckmann et al., 2010), and is also supported by our current and previous (Schuck et al., 2013a) data, which show that older adults' landmark-based spatial memory is less impaired than boundary-based memory. Second, the changed brain activation 
patterns are also consistent with predictions from a computational account of aging based on declined dopamine modulation ( $\mathrm{Li}$ et al., 2001; Li and Sikström, 2002). This account predicts that impaired dopamine functions in older adults lead to lower specificity of neural representations (dedifferentiation) and is supported by animal (Schmolesky et al., 2000) and human (Park et al., 2004) research. In support of this hypothesis, an analysis of the neural correlates of the landmark model with a more lenient threshold $(p<.005$, cluster size $=19$ ) showed that the activity for older adults extended into the thalamus and putamen (MNI: $-27 /-18 / 0$, see Fig. 5B). A corresponding control analysis for the boundary model did not show such a pattern (no younger adults' boundary related activity in the striatum and overall more focal brain activation patterns). Hence, our data suggest that neural representations during spatial navigation might be less specific to either the hippocampal or striatal systems, but instead involve both memory systems in older adults.

To further examine whether the additional recruitment of the hippocampal activity could be beneficial, we investigated whether the degree of dedifferentiation was related to performance. Specifically, we extracted contrast estimates of the boundary and landmark models in a (para)hippocampal and a striatum ROI separately for high and low performing younger and older adults (see 2.6). As can be seen in Fig. 5 (panels C and D), performance was indeed related to the modelpredicted activation in these areas. For the boundary model, only the high-performing younger adults showed a positive contrast in the (para)hippocampus ROI, whereas the low-performing younger adults did not. Interestingly, for the landmark model, high-performing older adults showed additional activation in the hippocampus ROI, whereas all older adults showed activation in the striatum ROI. Hence, among older adults, additional hippocampus function for landmark learning was related to superior learning performance. This finding is in line with the notion of a compensatory mechanism, such that additional hippocampal activity in older adults is related to carrying out 'striatal' functions and to enhanced landmark-based memory. Taken together, brain activities involved in spatial learning were less specific (or dedifferentiated) in older adults; at the same time, however, the additional recruitment of hippocampal activity helped performance.

In conclusion, the reported results showed that aging might change the links between hippocampal activity and the performed spatial memory function. Our study therefore offers a perspective on aging that highlights the potential of qualitative changes in brain-cognition links, rather than a sole focus on age-related impairments. Specifically, we found that while older adults' (para)hippocampal activity was related to landmark-based spatial memory, activity in the same area of younger adults was indicative of processing boundary information. A central challenge for future research is to better characterize such changes in functional anatomy of spatial navigation and to provide insight into the mechanisms that make such changes possible.

\section{Acknowledgments}

This study was conducted within the Neuromodulation of Lifespan Cognition Project, Centre for Lifespan Psychology, Max Planck Institute for Human Development and supported by the Max Planck Society, and by the Gottfried Wilhelm Leibniz Award 2013 of the German Research Foundation to UL. NWS was supported by the International Max Planck Research School LIFE. CFD is supported by the European Research Council (ERC-StG 261177) and the Netherlands Organisation for Scientific Research (NWO-Vidi 452-12-009). SCL's research is supported by the German Ministry for Education and Research (BMBF 01GQ091, 01GQ1313). We thank M Balt, H Hohl, M Maier, J Rodriguez and K Wermuth for their assistance with the data acquisition, B Steemers for his help with the virtual reality task, and $\mathrm{N}$ Bodammer for his help with setting up MRI sequences. We also thank F Thurm for stimulating discussions.

\section{Appendix A. Supplementary data}

Supplementary data to this article can be found online at http://dx. doi.org/10.1016/j.neuroimage.2015.05.031.

\section{References}

Andersson, J.L., Hutton, C., Ashburner, J., Turner, R., Friston, K., 2001. Modeling geometric deformations in EPI time series. Neuroimage 13, 903-919.

Ashburner, J., Friston, K.J., 2005. Unified segmentation. Neuroimage 26, 839-851.

Ashburner, J., Friston, K.J., 2009. Computing average shaped tissue probability templates. Neuroimage 45, 333-341.

Barnes, C.A., Suster, M.S., Shen, J., McNaughton, B.L., 1997. Multistability of cognitive maps in the hippocampus of old rats. Nature 388, 272-275.

Bird, C.M., Capponi, C., King, J.A., Doeller, C.F., Burgess, N., 2010. Establishing the boundaries: the hippocampal contribution to imagining scenes. J. Neurosci. 30, 11688-11695.

Blodgett, H., McCutchan, K., 1947. Place versus response learning in the simple T-maze. J. Exp. Psychol. 37, 412-422.

Burgess, N., O'Keefe, J., 1996. Neuronal computations underlying the firing of place cells and their role in navigation. Hippocampus 762, 749-762.

Chen, G., King, J.A., Burgess, N., O'Keefe, J., 2013. How vision and movement combine in the hippocampal place code. Proc. Natl. Acad. Sci. U. S. A. 110, 378-383.

Chowdhury, R., Guitart-Masip, M., Lambert, C., Dayan, P., Huys, Q., Düzel, E., Dolan, R.J., 2013. Dopamine restores reward prediction errors in old age. Nat. Neurosci. 16 , 648-653.

Daugherty, A.M., Yuan, P., Dahle, C.L., Bender, A.R., Yang, Y., Raz, N., 2014. Path complexity in virtual water maze navigation: differential associations with age, sex, and regional brain volume. Cereb. Cortex.

Devan, B.D., Goad, E.H., Petri, H.L., 1996. Dissociation of hippocampal and striatal contributions to spatial navigation in the water maze. Neurobiol. Learn. Mem. 66, 305-323.

Doeller, C.F., Burgess, N., 2008. Distinct error-correcting and incidental learning of location relative to landmarks and boundaries. Proc. Natl. Acad. Sci. U. S. A. 105, 5909-5914.

Doeller, C.F., King, J.A., Burgess, N., 2008. Parallel striatal and hippocampal systems for landmarks and boundaries in spatial memory. Proc. Natl. Acad. Sci. U. S. A. 105, 5915-5920.

Doeller, C.F., Barry, C., Burgess, N., 2010. Evidence for grid cells in a human memory network. Nature 463, 657-661.

Dombeck, D.A., Harvey, C.D., Tian, L., Looger, L.L., Tank, D.W., 2010. Functional imaging of hippocampal place cells at cellular resolution during virtual navigation. Nat. Neurosci. $13,1433-1440$

Domnisoru, C., Kinkhabwala, A.A., Tank, D.W., 2013. Membrane potential dynamics of grid cells. Nature 495, 199-204.

Eppinger, B., Schuck, N.W., Nystrom, L.E., Cohen, J.D., 2013. Reduced striatal responses to reward prediction errors in older compared with younger adults. J. Neurosci. 33, 9905-9912.

Etchamendy, N., Konishi, K., Pike, G.B., Marighetto, A., Bohbot, V.D., 2011. Evidence for a virtual human analog of a rodent relational memory task: a study of aging and fMRI in young adults. Hippocampus 880, 869-880.

Harris, M.A., Wiener, J.M., Wolbers, T., 2012. Aging specifically impairs switching to an allocentric navigational strategy. Front. Aging Neurosci. 4, 29.

Hartley, T., Trinkler, I., Burgess, N., 2004. Geometric determinants of human spatial memory. Cognition 94, 39-75.

Iaria, G., Palermo, L., Committeri, G., Barton, J.J.S., 2009. Age differences in the formation and use of cognitive maps. Behav. Brain Res. 192, 187-191.

Jezzard, P., Balaban, R.S., 1995. Correction for geometric distortion in echo planar images from B0 field variations. Magn. Reson. Med. 34, 65-73.

Konishi, K., Bohbot, V.D., 2013. Spatial navigational strategies correlate with gray matter in the hippocampus of healthy older adults tested in a virtual maze. Front. Aging Neurosci. 5,1

Lever, C., Burton, S., Jeewajee, A., O'Keefe, J., Burgess, N., 2009. Boundary vector cells in the subiculum of the hippocampal formation. J. Neurosci. 29, 9771-9777.

Li, S.-C., Lindenberger, U., Sikström, S., 2001. Aging cognition: from neuromodulation to representation. Trends Cogn. Sci. 5, 479-486.

Li, Sikström, 2002. Integrative neurocomputational perspectives on cognitive aging, neuromodulation, and representation. Neurosci. Biobehav. Rev.

Li, S.-C., Schmiedek, F., Huxhold, O., Rocke, C., Smith, J., Lindenberger, U., 2008. Working memory plasticity in old age: practice gain, transfer, and maintenance. Psychol. Aging 23, 731-742.

McDonald, R.J., White, N.M., 1994. Parallel information processing in the water maze: evidence for independent memory systems involving dorsal striatum and hippocampus. Behav. Neural Biol. 61, 260-270.

Moffat, S.D., 2009. Aging and spatial navigation: what do we know and where do we go? Neuropsychol. Rev. 19, 478-489.

Moffat, S.D., Resnick, S.M., 2002. Effects of age on virtual environment place navigation and allocentric cognitive mapping. Behav. Neurosci. 116, 851-859.

Moffat, S.D., Elkins, W., Resnick, S.M., 2006. Age differences in the neural systems supporting human allocentric spatial navigation. Neurobiol. Aging 27, 965-972.

Moffat, S.D., Kennedy, K.M., Rodrigue, K.M., Raz, N., 2007. Extrahippocampal contributions to age differences in human spatial navigation. Cereb. Cortex 17, 1274-1282.

Morcom, A.M., Good, C.D., Frackowiak, R.S.J., Rugg, M.D., 2003. Age effects on the neural correlates of successful memory encoding. Brain 126, 213-229.

Morris, R.G.M., Garrud, P., Rawlins, J.N.P., O'Keefe, J., 1982. Place navigation impaired in rats with hippocampal lesions. Nature 297, 681-683. 
Muller, R., Kubie, J., 1987. The effects of changes in the environment on the spatial firing of hippocampal complex-spike cells. J. Neurosci. 7.

Nagel, I.E., Preuschhof, C., Li, S.-C., Nyberg, L., Bäckman, L., Lindenberger, U., Heekeren, H.R., 2009. Performance level modulates adult age differences in brain activation during spatial working memory. Proc. Natl. Acad. Sci. U. S. A. 106, 22552-22557.

Newman, M.C., Kaszniak, A.W., 2000. Spatial memory and aging: performance on a human analog of the Morris Water Maze. Neuropsychol. Dev. Cogn. B Aging Neuropsychol. Cogn. 7, 86-93.

O'Keefe, J., Burgess, N., 1996. Geometric determinants of the place fields of hippocampal neurons. Nature 381, 425-428.

O'Keefe, J., Dostrovsky, J., 1971. The hippocampus as a spatial map. Preliminary evidence from unit activity in the freely-moving rat. Brain Res. 34, 171-175.

O'Keefe, J., Nadel, L., 1978. The hippocampus as a cognitive map. Oxford University Press, Oxford.

O'Keefe, J., Speakman, A., 1987. Single unit activity in the rat hippocampus during a spatial memory task. Exp. Brain Res. 68, 1-27.

Packard, M.G., McGaugh, J.L., 1992. Double dissociation of fornix and caudate nucleus lesions on acquisition of two water maze tasks: further evidence for multiple memory systems. Behav. Neurosci. 106, 439-446.

Packard, M.G., McGaugh, J.L., 1996. Inactivation of hippocampus or caudate nucleus with lidocaine differentially affects expression of place and response learning. Neurobiol. Learn. Mem. 65, 65-72.

Packard, M.G., Hirsh, R., White, N.M., 1989. Differential effects of fornix and caudate nucleus lesions on two radial maze tasks: evidence for multiple memory systems. J. Neurosci. 9, 1465-1472.

Park, D.C., Polk, T.A., Park, R., Minear, M., Savage, A., Smith, M.R., 2004. Aging reduces neural specialization in ventral visual cortex. Proc. Natl. Acad. Sci. U. S. A. 101, 13091-13095.

Poldrack, R.A., Fletcher, P.C., Henson, R.N., Worsley, K.J., Brett, M., Nichols, T.E., 2008. Guidelines for reporting an fMRI study. Neuroimage 40, 409-414.

R Development Core Team, 2011. R: A Language and Environment for Statistical Computing.

Raz, N., Lindenberger, U., Rodrigue, K.M., Kennedy, K.M., Head, D., Williamson, A., Dahle, C., Gerstorf, D., Acker, J.D., 2005. Regional brain changes in aging healthy adults: general trends, individual differences and modifiers. Cereb. Cortex 15, 1676-1689.

Restle, F., 1957. Discrimination of cues in mazes: a resolution of the "place-vs.-response" question. Psychol. Rev. 64, 217-228.

Reuter-Lorenz, P.A., Lustig, C., 2005. Brain aging: reorganizing discoveries about the aging mind. Curr. Opin. Neurobiol. 15, 245-251.
Rieckmann, A., Fischer, H., Bäckman, L., 2010. Activation in striatum and medial tempora lobe during sequence learning in younger and older adults: relations to performance. Neuroimage 50, 1303-1312.

Rodgers, M.K., Sindone, J.A., Moffat, S.D., 2012. Effects of age on navigation strategy. Neurobiol. Aging 33 (202.e15-e202.e22).

Rosenbaum, R.S., Winocur, G., Binns, M.A., Moscovitch, M., 2012. Remote spatial memory in aging: all is not lost. Front. Aging Neurosci. 4.

Schmidt-Hieber, C., Häusser, M., 2013. Cellular mechanisms of spatial navigation in the medial entorhinal cortex. Nat. Neurosci. 16, 325-331.

Schmolesky, M.T., Wang, Y., Pu, M., Leventhal, A.G., 2000. Degradation of stimulus selectivity of visual cortical cells in senescent rhesus monkeys. Nat. Neurosci. 3, 384-390.

Schneider, A., Huentelman, M.J., Kremerskothen, J., Duning, K., Spoelgen, R., Nikolich, K. 2010. KIBRA: a new gateway to learning and memory? Front. Aging Neurosci. 2, 4.

Schuck, N.W., 2013. Aging and Functional Reorganization of Striatum- and MedialTemporal Lobe-dependent Memory Systems (Doctoral dissertation), (Retrieved from http://edoc.hu-berlin.de/docviews/abstract.php?lang=ger\&id=40192).

Schuck, N.W., Doeller, C.F., Schjeide, B.M.M., Schröder, J., Frensch, P.A., Bertram, L., Li, S.C., 2013a. Aging and KIBRA/WWC1 genotype affect spatial memory processes in a virtual navigation task. Hippocampus 23, 919-930.

Schuck, N.W., Frensch, P.A., Schjeide, B.-M.M., Schröder, J., Bertram, L., Li, S.-C., 2013b. Effects of aging and dopamine genotypes on the emergence of explicit memory during sequence learning. Neuropsychologia 51, 2757-2769.

Solstad, T., Boccara, C.N., Kropff, E., Moser, M.-B., Moser, E.I., 2008. Representation of geometric borders in the entorhinal cortex. Science 322, 1865-1868.

Tolman, E.C., Ritchie, B.F., Kalish, D., 1946. Studies in spatial learning; place learning versus response learning. J. Exp. Psychol. 36, 221-229.

Tzourio-Mazoyer, N., Landeau, B., Papathanassiou, D., Crivello, F., Etard, O., Delcroix, N., Mazoyer, B., Joliot, M., 2002. Automated anatomical labeling of activations in SPM using a macroscopic anatomical parcellation of the MNI MRI single-subject brain. Neuroimage 15, 273-289.

Voermans, N.C., Petersson, K.M., Daudey, L., Weber, B., Van Spaendonck, K.P., Kremer, H.P.H., Fernández, G., 2004. Interaction between the human hippocampus and the caudate nucleus during route recognition. Neuron 43, 427-435.

Wiener, J.M., de Condappa, O., Harris, M.A., Wolbers, T., 2013. Maladaptive bias for extrahippocampal navigation strategies in aging humans. J. Neurosci. 33, 6012-6017.

Wilson, I.A., Ikonen, S., Mcmahan, R.W., Gallagher, M., Eichenbaum, H., Tanila, H., 2003. Place cell rigidity correlates with impaired spatial learning in aged rats. Annu. Rev. Psychol. 24, 297-305. 